\title{
Urteil des Hanseatischen Oberlandesgerichts vom Io. Juli 1977 (gegen Rechtsanwalt Kurt Groenewold)
}

\author{
Urteil \\ Im Namen des Volkes \\ In der Strafsache gegen \\ den Rechtsanwalt Kurt Reinhard Groenewold [...] hat der 3. Strafsenat des \\ Hanseatischen Oberlandesgerichts zu Hamburg auf die Hauptverhandlung vom 18. \\ Januar 1978 in der Sitzung vom 10. Juli 1978 [. . . ] für Recht erkannt: \\ Der Angeklagte wird wegen Unterstützung einer kriminellen Vereinigung in einem \\ besonders schweren Fall zu einer Freiheitsstrafe von \\ 2 (zwei) Jahren \\ verurteilt, deren Vollstreckung zur Bewährung ausgesetzt wird. \\ Der Angeklagte hat die Kosten des Verfahrens und seine notwendigen Auslagen zu \\ tragen. [...]
}

\section{Gründe}

$[\ldots]$

I. Der Angeklagte hat sich des fortgesetzten Unterstützens einer kriminellen Vereinigung in einem besonders schweren Fall nach $\$ I 29 Abs. I und Abs. 4 StGB schuldig gemacht.

Die in Haft befindlichen ehemaligen Mandanten des Angeklagten, die terroristischer Handlungen verdächtig waren, insbesondere Andreas Baader, Ulrike Meinhof, Gudrun Ensslin und Jan-Karl Raspe bildeten eine kriminelle Vereinigung im Sinne des $\$ r 29 Abs. I StGB.

Er unterstützte sie von Februar 1973 bis zu seinem Ausschluß vom »Stammheimverfahren « im Mai 1975.

Unter Vereinigung ist ein auf eine gewisse Dauer berechneter organisatorischer Zusammenschluß einer Anzahl von Personen zu verstehen, die bei Unterordnung des Willens des Einzelnen unter den Willen der Gesamtheit gemeinsame Zwecke verfolgen und unter sich derart in Verbindung stehen, daß sie sich als einheitlicher Verband fühlen (Urteil des BGH vom 21. Dezember 1975, BGHSt 27 Seite 325 ff.). Eine kriminelle Vereinigung liegt auch dann vor, wenn sich Personen zusammengeschlossen haben, die noch keine Straftaten begangen haben, aber die Begehung künftiger Straftaten ins Auge fassen. Die Absicht der Vereinigungen, Straftaten zu begehen, braucht noch nicht bis zur Vorbereitung einzelner Taten konkretisiert zu sein. Vielmehr reicht es aus, wenn sich die in der Vereinigung zusammengefaßten Mitglieder bewußt sind, daß es bei der Verfolgung ihrer Pläne zur Begehung erheblicher Straftaten kommen kann und daß sie dieses auch wollen.

Dieses liegt hier vor. Die ehemaligen Mandanten des Angeklagten haben sich "zum 
bewaffneten Kampf « verabredet, indem sie das »Konzept Stadtguerilla« verfolgten. Die ehemaligen Mandanten des Angeklagten beabsichtigten den Kampf gegen die Gesellschaft in der Bundesrepublik Deutschland nach dem Vorbild bisher begangener terroristischer Gewalttaten und nach dem Vorbild der südamerikanischen Stadtguerilla.

Dies räumt der Angeklagte ein. Er gibt zu, daß sich seine ehemaligen Mandanten als Vereinigung fühlten, wenn auch, wie er sagt, als »Prozeßvereinigung « und daß sie das »Konzept Stadtguerilla « vor Gericht vorbringen wollten und daß sie diesem Konzept anhängen. Dieses ergibt sich auch aus einer Vielzahl der zitierten Beiträge, die durch Vermittlung des Angeklagten im "Info-System « umgelaufen sind oder die der Angeklagte selbst wegen ihres bedenklichen Inhaltes zurückgehalten hat.

Die ehemaligen Mandanten des Angeklagten waren zum jederzeitigen bewaffneten Kampf gegen die Staatsmacht - in- und außerhalb der Haft - entschlossen. Etwaiger Mitgliederwechsel oder gar "Austritte « ändern daran nichts. Den Zusammenhalt der Gruppe zeigen die einheitlich durchgeführten Hungerstreiks, die Selbstkritiken und der Ausschluß aus der "gemeinschaftlichen Verteidigung « (Info I), wenn sich ein Gefangener der Disziplin und auch den Anweisungen zur Durchführung des Hungerstreiks nicht fügen wollte. Die Gruppenangehörigen hatten das Ziel, den einmal begonnenen terroristischen Kampf auch aus den Haftanstalten fortzuführen. Es handelt sich nicht nur um eine reine »Verteidigungsgemeinschaft $\ll$. Das Ziel der Gruppe der früheren Mandanten des Angeklagten ging darüber hinaus. Wie die Info-Beiträge zeigen, wollte man nicht nur ein "Selbstverständnis« als Motivierung für die Taten vor Gericht vorbringen, sondern gleichzeitig den Prozeß zum Kampfinstrument, zur Propagierung der auf die Zukunft gerichteten Ziele benutzen. Dies zeigen schon die Hungerstreikerklärung von Ulrike Meinhof und das provisorische Kampfprogramm. ${ }^{\text {I }}$ Dabei handelt es sich nicht mehr um Erklärungen für ein vergangenes Tun oder um eine Auseinandersetzung mit der Anklageschrift, sondern auch um den Aufruf zur künftigen »Revolutionierung «. Auch die im Stammheimprozeß eingebrachten, hier zitierten Beweisanträge ${ }^{2}$ machen deutlich, daß neben echter Verteidigung auch staatsfeindliche Aktivität gewollt war, wie der Inhalt und die Wortwahl deutlich zeigen.

$\mathrm{Daß}$ über reine Verteidigung hinaus in die Zukunft weisend revolutionäre Ziele und damit auch Straftaten beabsichtigt waren, zeigen die Hungerstreikerklärung von Ulrike Meinhof und das gleichzeitig veröffentlichte provisorische Kampfprogramm. Der Info-Beitrag So $23 \mathrm{I}$ I/2.4-30 zeigt ebenfalls, daß über eine Selbstorganisation der sog. politischen Gefangenen die Massen für die Revolution gewonnen werden sollten. Die Vielzahl der im Urteil zitierten Beiträge, seien sie umgelaufen, seien sie von dem Angeklagten zurückgehalten worden, machen deutlich, daß über Verteidigungsvorbereitungen hinaus - also Material zur Offenlegung der Motive für die angeklagten Taten - eine in die Zukunft weisende Diskussion zur Aufrechterhaltung des »Konzeptes Stadtguerilla« gewollt war.

2. Der Angeklagte hat die kriminelle Vereinigung unterstützt. Er hat die kriminelle Organisation in ihren Bestrebungen und in ihrer Tätigkeit gefördert und dadurch bestärkt, daß er in dem von ihm betriebenen »Info-System« Beiträge umlaufen ließ,

\footnotetext{
I Die "Hungerstreikerklärung « und das "Provisorische Kampforogramm für den Kampf um die politischen Rechte der gefangenen Arbeiter" wurde von Ulrike Meinhof am 13. 9. 1974 im Berliner Mahler-Prozeß verlesen; der Text wurde als Flugschrift verbreitet und ist nachgedruckt in: Komitees gegen Folter an politischen Gefangenen in der BRD, Hrsg., Der Kampf gegen die Vernichtungshaft, o. O. u. o. J. (1974).

2 Beweisantrag von Andreas Baader vom 29. 3. 1977, abgedruckt in: Ulf G. Stuberger, Hrsg., "In der Strafsache gegen Andreas Baader, Ulrike Meinhof, Jan-Carl Raspe, Gudrun Ensslin wegen Mordes u. a." Dokumente aus dem Prozeß, Frankfurt am Main 1977, S. $263 \mathrm{ff}$.
} 
die nicht mehr Verteidigungszwecken dienten, sondern für eine gegenseitige Unterrichtung und Schulung bestimmt waren und die dazu beitrugen, daß die Mitglieder der kriminellen Vereinigung in ihren kriminellen Zielen bestärkt wurden und weiter daran festhielten. Der Angeklagte unterstützte die kriminelle Vereinigung weiterhin dadurch, daß er in seinen Verteidigerrundbriefen Anweisungen von Andreas Baader in bezug auf den Hungerstreik weitergab. Gerade die Anweisungen zu einem bestimmten Verhalten im Hungerstreik, durch das der Tod einiger Gefangener in Kauf genommen wird, verstärkte den Gruppenzusammenhalt in besonderer Weise. Als Unterstützung einer kriminellen Vereinigung genügt es, wenn diese Organisation in ihren Bestrebungen gefördert und insbesondere bestärkt wird (Urteil des BGH vom 30. Oktober 1964 in BGHSt Band 20 Seite 89 f.).

$\mathrm{Da}$ die früheren Mandanten des Angeklagten gemeinschaftlich angeklagt worden waren und Verteidiger ihnen so beigeordnet worden waren, daß praktisch jeder Verteidiger im Stammheim-Verfahren jeden der dort Angeklagten verteidigte, war eine gegenseitige Unterrichtung zum damaligen Zeitpunkt eine erlaubte Verteidigungshandlung. Es war zulässig, daß Beiträge zwischen den Gefangenen umliefen, in denen sie sich mit Material beschäftigten, das der Darlegung der Motivationslage für die damals angeklagten Taten diente. Es war auch zulässig, Beiträge weiterzugeben, in denen sich die Gefangenen über ihre Ansichten äußerten, soweit nicht in ihnen auf die Zukunft hinweisend revolutionärer Umsturz und bewaffneter Kampf - damit also Mord, Sprengstoffversprechen und Geiselnahme - befürwortet wurden. Zulässig war es sicher auch, daß Beiträge umliefen, in denen man sich mit der Organisation von staatlichen Institutionen zur Zeit der angeklagten Taten beschäftigte, da dies der Darlegung der Motivation für diese dienen kann. Schließlich wäre es nicht zu beanstanden, wenn Beiträge weitergegeben worden wären, in denen man sich mit von der Anklagebehörde vorgelegten Gutachten auseinandergesetzt hätte. Im Rahmen solcher zulässiger Umläufe ist es hinzunehmen, wenn dadurch automatisch ein gewisses Zusammengehörigkeitsgefühl der ehemaligen Mandanten des Angeklagten bestärkt und wenn sie in ihrer kriminellen Haltung aufrechterhalten werden. Dies folgt aus der in der damaligen Zeit zulässigen gemeinschaftlichen Verteidigung.

Im Rahmen der gemeinschaftlichen Verteidigung war es auch zulässig, daß der Angeklagte selbst Rundbriefe in der geschilderten Art umlaufen ließ, in denen über das gegenseitige Ergehen während des Hungerstreiks, über Verteidigungstaktik, über die Reaktionen in der Öffentlichkeit und über Gerichtsbeschlüsse und Verfahren berichtet wurde.

Eine Unzahl von Beiträgen, die über das Info-System liefen und eine Vielzahl von Verteidigerbriefen fallen darunter. Sie können nicht als strafbare Unterstützung einer kriminellen Vereinigung angesehen werden.

Folgende Beiträge, die durch das "Info-System « liefen, und folgende Rundbriefe des Angeklagten dienen jedenfalls nicht mehr der Verteidigung, sondern der Aufrechterhaltung und Förderung der kriminellen Vereinigung. Sie können auch bei weitgehender Auslegung nicht mehr als Verteidigungshandlungen angesehen werden:

Dazu gehört die Hungerstreikerklärung und das vorläufige Kampfprogramm von Ulrike Meinhof, die der Angeklagte in seinem Info-System verbreiten ließ. Die Propagierung von proletarischer Gegengewalt und des Klassenkampfes (- Freiheit durch bewaffneten antiimperialistischen Kampf -) hat nichts mit Verteidigung zu tun. Sie ist auf die Zukunft gerichtet. Die Weitergabe dieses Schriftstückes auch als Anlage zu Info-Beiträgen unterstützte die kriminelle Vereinigung in ihrem Selbstverständnis als "Stadtguerilla« und dient, wie auch die anderen Beiträge zeigen, in denen sie erwähnt wird, der ideologischen Untermauerung. 
Auch die Beiträge So $23 \mathrm{I} \mathrm{I/2.4-30;} \mathrm{So} 35 \mathrm{~T} / \mathrm{I} / \mathrm{I}$ Bleistiftnummer 48 ; So $35 \mathrm{~T} \mathrm{I/I-I}$ und So $35 \mathrm{~T} / \mathrm{I} / 2-\mathrm{I} 2$ (Zellenplan); und So $35 \mathrm{~T} \mathrm{2/2/5-163}$ weisen in die Zukunft und dienen nicht der Verteidigung, sondern nur noch dem Selbstverständnis der ehemaligen Mandanten als "Stadtguerilla «. Der Angeklagte kann sich nicht darauf berufen, es sei seine Pflicht als Anwalt, gerade dieses Selbstverständnis aufrechtzuerhalten, da seine Mandanten sonst als Menschen zerbrechen würden und dieses Selbstverständnis nicht vor Gericht darstellen könnten. Es ist weder Pflicht noch Recht eines Anwalts, ein Selbstverständnis und eine politische Identität zu unterstützen und aufrechtzuerhalten, die verfassungsfeindlich und auf künftige Gewalt gerichtet ist. Die Darstellung eines solchen Selbstverständnisses vor Gericht ist keine Verteidigung, sondern dient nur der Umfunktionierung eines Prozesses in staatsfeindliche Propaganda und Aufruf zur Gewalt.

Als unabhängiges Organ der Rechtspflege soll der Verteidiger mithelfen, das Recht zu verwirklichen. Damit tritt er an die Seite der Gerichte und der Staatsanwaltschaft. Auf diese hat der Rechtsanwalt Bedacht zu nehmen und jedenfalls nicht den Kampf seiner Mandanten gegen diese Institutionen zu unterstützen (vgl. Beschluß des BGH vom I3. Februar 1978 AnwZ (B) $14 / 77$, dort auch weitere Hinweise). Dem Angeklagten ist zuzugeben, daß er nicht verpflichtet ist, gegen das "Selbstverständnis« seiner Mandanten einzuschreiten und ihr Zensor zu sein. Er durfte sie aber nicht durch Weiterleitung ihrer »Selbstverständnispapiere« unterstützen.

Ebenso gehört die Weitergabe der Beiträge, die sich mit Sprengtechnik, Bomben, Polizei und Grenzschutz beschäftigen, nicht mehr zur Verteidigung. Dazu gehören die Beiträge: So $30 \mathrm{I} / 6 / 3.3 / 2$ (Elektronik-Meßwandler); So 30 Seite $18 \mathrm{I} / 6 / 3.3 / 3$ (Sender Tirana); So 29 I I/4.4-3 (Polizeiorganisation und Schwerpunkte der Polizei); So 29 Blatt $385-387$ RS (Verfassungsschutzbericht und Zeichnungen von Bomben und Raketen) sowie der Beitrag $\mathrm{SH}_{4}$ Blatt 88 (Feder und das Gewehr. Für die Revolution!!!)4.

Diese Beiträge können auch bei großzügiger Auslegung nicht mehr als zur Vorbereitung der Verteidigung dienlich angesehen werden. Sie enthalten keine Auseinandersetzung mit Anklagepunkten, sondern sind reine Schulungspapiere zur Vorbereitung künftiger Gewaltakte.

Schließlich dient zumindest auch die Weitergabe der Anweisungen Andreas Baaders zum Hungerstreik (So 35 T/2/2/8 Bleistiftnummern 27 und 28 und So s Blatt 19)'

3 Bei diesen Texten handelt es sich um einen "Grundriß zur Gefangenenorganisation, in dem auf das "Kampfprogramm « Bezug und als Ziel »das prinzip revolutionärer Organisation von den massen zu den massen « proklamiert und von einer "aufbauphase " gesprochen wird "bis die organisation in den gefängnissen ein niveau erreicht hat (politisch, organisatorisch, praktisch) von dem aus sie kriterien für bündnisse, aktion draußen propaganda usw. festlegt «; ferner um einen Brief von Carmen Roll, in dem es heißt: »mut - dir das bewußtsein der illegalität des kampfes erobern. [ . . ] die fahndungsbehörden haben nunmehr zur kenntnis zu nehmen, daß sie keinen von uns liquidieren können, ohne damit rechnen zu müssen, daß wir zurückschlagen werden"; in einem anderen Text heißt es: "trotzdem war es [der Anschlag auf die Botschaft in Stockholm] keine niederlage, es war die stärkste antiimperialistische aktion des deutschen proletariats überhaupt. [...] stockholm war das dien bien phu der revolution in deutschland, das stalingrad des neuen faschismus, das danang der revolution in westeuropa. - der durchbruch «; im letzten der erwähnten texte ebenfalls vom Stockholmer Anschlag die Rede: "sie [die Aktion] ist eine niederlage - aber wie che weiß: die hauptsache ist in niederlagen + siegen die kontinuität des angriffs, darüber wirkt die guerilla, das ist das prinzip dieser phase."

${ }_{4}$ Bei diesen Texten handelt es sich um eine Ausarbeitung, die mit "Elektronik-Meßwandel/Schalter" überschrieben ist und bei der es »um die umwandlung nichtelektrischer physikalischer werte in elektrische " geht; sodann um eine Anzeige für ein bestimmtes Kofferradio auf der Angaben darüber notiert waren, wie und wann »donnerstags: aus der front des befreiungskampfes der völker « des Senders Tirana empfangen werden kann; um Angaben über die Aufwendungen des Landes NRW für die Polizei, über die Aufstellung von Gruppen mit "präzisionsschützen « und mobile Einsatzgruppen etc.; um den Verfassungsschutzbericht 1973 (Auszüge) sowie um ein Schreiben, das in meiner Anmerkung zum Urteil zitiert wird; "mao schreibt ...."

s In diesem Text von Andreas Baader heißt es: »befehl ist [ . . ] es gibt nur eine situation in der wir damit 
nicht mehr der Verteidigung oder einer berechtigten Sorge um die Gesundheit seiner Mandanten. Es handelt sich vielmehr um einen Beitrag, der der Förderung des Gruppenzusammenhaltes dient. Durch die Aussicht auf den Tod und durch die Bereitschaft, sein Leben für die Gruppe einzusetzen oder für diese zu leiden, wird ein starkes Zusammengehörigkeitsgefühl erzeugt. Dieses Zusammengehörigkeitsgefühl sollte - wie die Beiträge zeigen - unter evtl. Schaffung von Märtyrern zum bewaffneten Kampf eingesetzt werden. Dazu trug der Angeklagte durch die Weitergabe dieser Befehle bei.

Auch durch die Versendung eigener Rundschreiben hat der Angeklagte die kriminelle Vereinigung unterstützt.

Wenn auch eine Unterstützung des Hungerstreiks und die Weitergabe von Weisungen zum Beginn oder Abbruch desselben und hinsichtlich eines bestimmten Aussageverhaltens (Gesagt wird nichts) zumindest noch kein strafbares Verhalten ist, so stellt doch die Ubernahme von Baaders Standpunkt in dem Rundschreiben vom I5. Oktober 1974 (So $38 \mathrm{Nr}$. Io: Zielsetzung, Hungerstreik auch wenn einer stirbt. Kampfprogramm wird vorbereitet und überregional verteilt) $)^{6}$ die Unterstützung einer kriminellen Vereinigung dar. Das revolutionäre Gruppenbewußtsein wird durch den Hinweis auf den Tod und den bewaffneten Kampf (siehe Erklärung von Ulrike Meinhof) gestärkt. Dies gilt auch für die kritiklose Weitergabe von Werner Hoppes Meinung im Schreiben vom 3. Juli 1973 (So I Blatt 31-36) ${ }^{7}$ mit der Aufforderung - nur so kann es verstanden werden -, Gewalt gegen Richter anzuwenden. Es ist auch nicht zu übersehen, daß die Übernahme der Wortwahl der Gefangenen durch den Angeklagten (Isolationsfolter, Vernichtungshaft, Bullen, Schwein etc.) in diesem Zusammenhang geeignet ist, diese in ihrer Gesinnung zu bestärken und zu unterstützen, da sie sich in ihrer Haltung und Ansicht nicht alleingelassen fühlen.

3. Es liegt auch ein besonders schwerer Fall im Sinne des $\ 129$ Abs. 4 StGB vor. Bei der kriminellen Vereinigung, die die ehemaligen Mandanten des Angeklagten (Andreas Baader, Gudrun Ensslin, Ulrike Meinhof etc.) bildeten, handelt es sich um eine schwer kriminelle Vereinigung, deren Ziele die Begehung von Sprengstoffanschlägen u. a. einschloß. Nach nunmehr geltendem Recht läge eine terroristische Vereinigung nach $\$ 129a StGB vor. Bei der Betätigung für eine solche schwer kriminelle Vereinigung ist nach dem Rechtszustand vor Inkrafttreten des $\$ I 29a StGB in der Regel von einem besonders schweren Fall im Sinne des $\$ I 29 Abs. $4 \mathrm{StGB}$ auszugehen (Urteil des BGH vom 14. September 1977, MDR 1978 Seite 63 f.).

Der Senat sieht keinen Anlaß, von diesem Regelfall abzuweichen. Gerade die zahlreichen Umläufe (Elektronik-Meßwandler) und andere, auch vom Angeklagten zurückgehaltene Beiträge (Sprengstoffbunker) zeigen, daß es sich um eine Vereini-

\footnotetext{
einverstanden sind - wenn unter dem druck akuter lebensgefahr für dich die isolation für alle aufgehoben wird [ . . ] klar ist - muß sein, daß die anwälte ne andere linie (+ nicht erst jetzt) bekommen haben. sie kämpfen um unser leben [.. .] die verlegung in eine klinik für alle ist keine forderung der anwälte. es gibt nur eine forderung: die vollständige aufhebung der isolation - sonderbehandlung -." Im zweiten der erwähnten Texte schreibt Baader: "man muß sich darüber klar sein, daß bei diesem hungerstreik einer oder zwei sterben können - aber nicht mehr + die wirkung wird dann sowieso die lage aller verändern«.

6 Bei diesem Text handelt es sich um einen Brief von RA Groenewold vom I 5. 10. 1974. In diesem Brief heißt es: "Nocheinmal: Niemand soll sich gegen Zwangsernährung wehren (es sei denn, einheitlich). [...] Ob falsch oder richtig, Zielsetzung, Hungerstreik auch wenn einer stirbt. Die politisch richtige Zielsetzung ist Hungerstreik bis zur Aufhebung der Sonderbehandlung und Gleichstellung mit anderen Gefangenen."

7 Der Text lautet: "was die teilnahme an prozessen betrifft, so finde ich es richtig, nicht mehr hinzugehen das argument, das evtl. dagegen spricht, nämlich mal ein schwein dabei voll zu treffen, wird dadurch aufgehoben, daß sie ja erstmal sowieso versuchen, einen mit gewalt hinzuschleppen, und wenn es so eine möglichkeit gibt, dann läßt sie sich dann auch nutzen - wie in karlsruhe kürzlich ursel versuchte.«
} 
gung handelt, die schwer kriminell war und daß der Angeklagte dies auch wußte.

4. Der Angeklagte handelte vorsätzlich. Er gab die erwähnten Beiträge und Schreiben in Kenntnis ihres Inhaltes wie geschildert weiter. Für die Annahme eines Irrtums über Tatsachen besteht kein Anhaltspunkt.

5. Der Angeklagte handelte auch rechtswidrig. Einen Rechtfertigungsgrund der Verteidigung gibt es für das festgestellte Verhalten nicht. Es ist bereits festgestellt worden, daß das ihm vorgeworfene Verhalten keine Verteidigung war, soweit er die Grenzen des Zulässigen überschritten hat.

6. Der Angeklagte war der Ansicht, sein Verhalten sei erlaubte Verteidigung. Er irrte in dieser Hinsicht. Der Irrtum war vermeidbar ( $\$$ I7 StGB).

Der Angeklagte handelte grob rechtsfahrlässig. Bei gewissenhafter Prüfung hätte er erkennen müssen und auch erkennen können, daß er die Grenzen zulässiger Verteidigung weit überschritt. Der Angeklagte rechnete zumindest damit, daß seine Mandanten schon vor ihrer Verhaftung eine kriminelle Vereinigung bildeten, wie er selbst in der Hauptverhandlung zugab. Als ihr Verteidiger wußte er, was ihnen vorgeworfen wurde. Er kannte ihre Ansichten. Bei der Lektüre der Beiträge, die er weiterleitete, hat er erkannt, daß sie auf künftige Guerillaaktivitäten abstellten und entsprechende Kenntnisse und Empfehlungen vermittelten und dennoch möglicherweise geglaubt, sie im Rahmen seiner Verteidigertätigkeit weiterleiten zu dürfen. Er hätte er kennen können, daß ein Bezug zu Gutachten oder zu Anklagevorwürfen in ihnen nicht enthalten war und daß sie in die Zukunft und auf zukünftige Aktionen hinwiesen. Die ganzen vorher aufgeführten Punkte, die gegen einen Irrtum des Angeklagten sprechen könnten, zeigen, daß der Angeklagte diesen Irrtum vermeiden konnte. Daß andere Rechtsanwälte dem gleichen Irrtum unterlagen, ändert daran nichts. Es gab sicher Gesinnungsgenossen des Angeklagten, die ebenso weit wie er selbst gingen. Das mußte er sich aber auch sagen.

Der Angeklagte kann sich nicht darauf berufen, daß er bei Erkundigungen bei den zuständigen Stellen (Anwaltskammer) keine befriedigenden Auskünfte bekommen hätte. Er selbst hätte bei richtiger Úberlegung, bei Vorsicht und Sorgfalt und bei Beurteilung des offen zutage tretenden Gruppenzusammenhalts und der staats- und verfassungsfeindlichen Gesinnung erkennen müssen, daß er bei allem Recht und bei aller Pflicht zur Verteidigung diesen Zusammenhalt und diese Gesinnung, die auf künftigen Terror gerichtet war, nicht hätte unterstützen dürfen.

Der Angeklagte kann sich auch nicht auf angebliche ausländische Rechtsordnungen berufen, nach denen sein Verhalten erlaubt wäre. Nach $₫ 33$ StGB gilt das deutsche Strafrecht für Taten, die im Inland begangen werden. Das ist hier der Fall. Der Angeklagte als deutscher Rechtsanwalt hat sich in Deutschland strafbar gemacht, deutsches Recht ist anzuwenden. Ein verbindliches Völkerrecht über die Rechte der Verteidigung in politischen Prozessen gibt es nicht, ebensowenig wie ein innereuropäisches Strafprozeßrecht, nach dem das Verhalten des Angeklagten erlaubt wäre. Die Vernehmung der vom Angeklagten sistierten Zeugen hat im übrigen auch keinen Anhaltspunkt ergeben, daß irgendeine ausländische Rechtsordnung es den Verteidigern gestattet, im Rahmen ihrer Privilegien unter inhaftierten Guerilla Hinweise darüber zu erteilen, wie man künftig am besten bewaffnete Polizisten ortet oder die Entschärfung von Sprengmitteln verhindert oder verzögert.

Der Angeklagte kann sich auch nicht darauf berufen, daß ihm ausländische Rechtsanwälte sagten, sein Verhalten entspräche internationalen Gepflogenheiten. Als deutscher Rechtsanwalt hatte er sich nach deutschem Recht zu richten.

Da der Angeklagte den Irrtum vermeiden konnte, handelte er schuldhaft. [...]

I. Bei der Strafzumessung hat der Senat von der Milderungsmöglichkeit nach $\$ \$ 17$ 
Satz 2, 49 Abs. I StGB Gebrauch gemacht. Es war infolgedessen von einem Strafrahmen von einem Monat bis zu drei Jahren und neun Monaten auszugehen. Strafschärfend wurde über die Annahme eines besonders schweren Falles hinaus berücksichtigt, daß die Gruppe, die der Angeklagte unterstützte, außerordentlich gefährlich war, wie schon die Beiträge zeigen, und daß sie eine erschreckende Militanz zeigte. Der Angeklagte unterstützte diese Organisation über eine längere Zeit, und zwar von 1973 bis 1975 . Auch nach Bekanntwerden der Befreiungspläne Anfang 1974 setzte der Angeklagte seine Unterstützungshandlungen fort. Weiterhin mußte strafschärfend berücksichtigt werden, daß der Angeklagte bei der Unterstützung außerordentlich aktiv vorging. Seine massive Unterstützung hat den Zusammenhalt seiner ehemaligen Mandanten und ihre Ziele ganz wesentlich gefördert. Der Angeklagte hat damit eine beachtliche Gefährdung der Offentlichkeit für den Fall einer Freilassung oder Freipressung der Gruppe in Kauf genommen.

Mildernd hat der Senat berücksichtigt, daß der Angeklagte bereit war, mit der Verteidigung seiner ehemaligen Mandanten eine erhebliche Verantwortung auf sich zu nehmen. Die Úbernahme der Verteidigung der in der Offentlichkeit bereits vorverurteilten Baader-Meinhof-Gruppe, die ein Recht darauf hatte, verteidigt zu werden, war grundsätzlich ehrenwert. Der Angeklagte zeigte sich als ein einsatzfreudiger, engagierter Rechtsanwalt. Er suchte neue Wege und bemühte sich, Denkanstöße zu vermitteln. Es ist auch durchaus achtenswert, daß er bei der öffentlichen Vorverurteilung seiner Mandanten und in der gespannten Atmosphäre immer wieder darauf hinwies, daß seine Mandanten noch nicht verurteilt seien und $\mathrm{da} ß$ auch sie Anspruch auf ein faires, objektives Verfahren hätten. Der Senat konnte es auch nicht unberücksichtigt lassen, daß die Beiträge, deren Verbreitung als strafbare Unterstützung einer kriminellen Vereinigung gewürdigt wurden, nur ein geringer Teil der Schriftwerke sind, die der Angeklagte zur Verteidigung seiner Mandanten erlaubtermaßen verteilt hat. Von diesem gewaltigen Einsatz des Angeklagten für seine Mandanten war der Großteil rechtmäßig.

In der Hauptverhandlung fand mit dem Angeklagten ein offenes, freimütiges Rechtsgespräch statt. Den objektiven Sachverhalt hat er eingeräumt. Er gestand zu, daß er in einigen Punkten doch zu weit gegangen sei, und er erklärte weiterhin, daß er jetzt doch vieles anders machen würde. Auch dies hat der Senat strafmildernd berücksichtigt.

Schließlich war zu bedenken, daß sich der Angeklagte in der Hauptverhandlung von den Ansichten seiner ehemaligen Mandanten deutlich distanziert hat, sich als bürgerlichen Anwalt bezeichnet hatte und daß ein Kontakt zwischen ihm und der "Anarchistenszene «, wie ihm zu glauben ist, seit Jahren nicht mehr besteht.

Unter Berücksichtigung aller dieser Punkte hält der Senat eine Freiheitsstrafe von zwei Jahren für angemessen.

2. Der Senat hat die Vollstreckung dieser Freiheitsstrafe gemäß $\ 56$ Abs. 2 StGB zur Bewährung ausgesetzt.

Es liegen besondere Umstände in der Person und in der Tat des Angeklagten vor, die dies rechtfertigen.

Der Angeklagte ist nicht vorbestraft. Er befand sich bei der Verteidigung seiner ehemaligen Mandanten in einer Ausnahmesituation. Er sah, daß deren Verurteilung von der Öffentlichkeit und den Massenmedien gefordert wurde und fühlte sich, um diesem Verurteilungsdruck zu begegnen, mit Recht zu besonderer Aktivität veranlaßt. Er sah weiterhin, daß sich viele seiner Mandanten in einer sehr schlechten körperlichen Verfassung befanden, und wurde auch aus Sorge um ihre Person tätig, wobei er allerdings weit über das Notwendige hinausging. Dennoch kann nicht übersehen werden, daß der Angeklagte aus nicht verachtenswerten Gründen han- 
delte. Schließlich befand sich der Angeklagte in einem wenn auch vermeidbaren Irrtum - wie nicht auszuschließen ist -, über die Grenzen dessen, was noch Verteidigungshandlung und was Unterstützungshandlung ist.

Auch besondere Umstände in der Tat sprechen für eine Strafaussetzung zur Bewährung. Es handelte sich um eine außergewöhnlich umfangreiche Verteidigung gegen eine schwerwiegende Anklage. Durch das Oberlandesgericht Stuttgart sind den Angeklagten, wie schon dargestellt, ihre Verteidiger beigeordnet worden, so daß jeder Verteidiger jeden Angeklagten vertrat. Die gemeinschaftliche Verteidigung und eine gegenseitige Unterrichtung waren demnach erlaubt. Im Rahmen dieses erlaubten Informationsflusses kam es zu den Unterstützungshandlungen durch den Angeklagten. Viele der umgelaufenen Beiträge beruhen auf allgemein zugänglichen Quellen, die die ehemaligen Mandanten des Angeklagten auf legale Weise nach Passieren der Zensur erhielten.

Es ist mit nahezu völliger Sicherheit zu erwarten, daß der Angeklagte sich schon die Verurteilung zur Warnung dienen lassen und künftig keine Straftaten mehr begehen wird. Der Angeklagte steht den Mandanten und Aktivitäten der damaligen Zeit seit Jahren fern. Wegen der veränderten Gesetzeslage ( $\$$ I46 StPO) ist die Tat des Angeklagten in solcher Ausgestaltung nicht mehr wiederholbar im Unterschied zu fast allen anderen Straftaten.

Unter Berücksichtigung dieser Punkte ist der Senat der Ansicht, daß hier mehr als nur »durchschnittliche« Strafmilderungsgründe eine Aussetzung der Vollstreckung der Freiheitsstrafe rechtfertigen. Es liegen vielmehr mildernde Umstände von besonderem Gewicht vor, die Ausnahmecharakter haben. Die Tat des Angeklagten ist außergewöhnlich (Urteil des BGH vom I 3. Januar 1977, I StR 691/76).

Gemäß $\ 56 \mathrm{~b}$ Abs. 2 Ziffer 2 StGB hat der Senat dem Angeklagten auferlegt, einen Geldbetrag von $75000,-$ DM in monatlichen Raten von $5000,-$ DM zugunsten des Witwen- und Waisenfonds der Polizei Hamburg zu zahlen. Diese Auflage ist zur Genugtuung für das begangene Unrecht notwendig. Unter Berücksichtigung der von dem Angeklagten begangenen Tat und unter Berücksichtigung seines nicht unerheblichen Vermögens ist ihm diese Zahlung auch zumutbar.

$[\ldots]$

Der Senat hat gegen den Angeklagten kein Berufsverbot verhängt ( $\$ 70$ Abs. I $\mathrm{StGB})$.

Eine Gesamtwürdigung des Angeklagten und seiner Tat läßt nicht die Gefahr erkennen, daß er bei weiterer Ausübung seines Berufes erhebliche rechtswidrige Taten begehen wird, wie sie hier festgestellt worden sind. Der Senat hat schon darauf hingewiesen, daß diese Tat praktisch nicht wiederholbar ist. Der Angeklagte steht seit 1975 nicht mehr mit seinem früheren Mandantenkreis oder mit Mandanten ähnlicher Art in Verbindung. Für ein Berufsverbot spricht auch nicht, daß der Angeklagte zum Teil recht scharfe Veröffentlichungen zu diesem Prozeß vor Verfahrensbeginn publiziert hat. [...]

$\mathrm{Ob}$ gegen den Angeklagten im ehrengerichtlichen Verfahren ein Berufsverbot, dessen Voraussetzungen standesrechtlich anders gelagert sind, ausgesprochen bzw. sein Ausschluß aus der Anwaltschaft ausgesprochen werden muß, hatte das Gericht nicht zu beachten.

$[\ldots]$

Dr. Plambeck

Bürrig

v. Heppe

Först

Dr. Meister 